\title{
A formal analysis of some factor- and precedent-based accounts of precedential constraint
}

\author{
Henry Prakken ${ }^{1,2}$ (D)
}

Accepted: 21 January 2021 / Published online: 23 February 2021

(C) The Author(s) 2021

\begin{abstract}
In this paper several recent factor- and dimension-based models of precedential constraint are formally investigated and an alternative dimension-based model is proposed. Simple factor- and dimension-based syntactic criteria are identified for checking whether a decision in a new case is forced, in terms of the relevant differences between a precedent and a new case, and the difference between absence of factors and negated factors in factor-based models is investigated. Then Horty's and Rigoni's recent dimension-based models of precedential constraint are critically examined. An alternative to their reason models is proposed which is less expressive but arguably easier to apply in practice.
\end{abstract}

Keywords Case-based reasoning $\cdot$ Precedential constraint $\cdot$ Factors $\cdot$ Dimensions

\section{Introduction}

Reasoning with cases has long been an important topic in the computational study of legal argument. When interpreting general legal terms in a new case, the legal rules often 'run out' (Gardner 1987) and judges and lawyers resort to precedents in which these terms were applied to concrete cases. Since the new case often does not fully match the past cases, reasoning with precedents is distinct from reasoning with legal rules and involves drawing analogies to past cases and discussing their relevant similarities and differences. Starting with the seminal work on the HYPO system for US trade secrets law (Rissland and Ashley 1987), much AI \& Law research on casebased reasoning represents cases as sets of case features. A case feature is a legally relevant fact pattern which tends to favour one side or the other. Feature sets have to be weighed or balanced in each case, unlike legal rules, of which the conditions

Henry Prakken

h.prakken@uu.nl

1 Department of Information and Computing Sciences, Utrecht University, Utrecht, The Netherlands

2 Faculty of Law, University of Groningen, Groningen, The Netherlands 
are ordinarily sufficient for accepting their conclusion. Features can be boolean (e.g. 'the secret was obtained by deceiving the plaintiff', 'a non-disclosure agreement was signed' or 'the product was reverse-engineerable'), in which case they are called factors. Features can also be multi-valued (e.g. the number of people to whom the plaintiff had disclosed the secret or the severity of security measures taken by the plaintiff), in which case they are called dimensions.

The first AI \& law studies of factor- and dimension-based reasoning were not formal but design- and application-oriented. Moreover, the designed systems (HYPO, Rissland and Ashley 1987; Ashley 1990 and CATO, Aleven and Ashley 1991; Aleven 1997) were primarily rhetorical in nature in that they did not compute an 'outcome' or 'winner' of a dispute but instead generated debates as they can take place between 'good' lawyers. Later work was more logic- and outcome-oriented (Loui et al. 1993; Hage 1993; Prakken and Sartor 1998). The present paper ${ }^{1}$ is in the latter tradition. It particularly contributes to a line of research initiated by Horty (2011), which aims to formalise the common-law concept of precedential constraint, that is, to characterise the conditions under which a decision in a new case is forced or at least allowed by a body of precedents. This is a problem not addressed in the initial work on the HYPO and CATO systems. Initially Horty only studied factor-based reasoning but recently he has adapted his approach to dimensions (Horty 2017, 2019, 2020). The relevance of this line of research is not confined to common-law jurisdictions since the question what a body of precedents says about a particular legal problem can also arise in civil-law jurisdictions (although possibly in different ways; see e.g. Ashley (2004)).

In this paper I present four main contributions to the line of research initiated by Horty. First, I will identify simple syntactic criteria for checking whether a decision in a new case is forced, in terms of the relevant differences between a precedent and a new case. I will study such criteria for both factor- and dimension-based models of precedential constraint. Second, I will investigate to which extent the difference between absence of factors and negated factors is relevant in Horty's factor-based models of precedential constraint. Third, I will present a full formalisation of Rigoni's (2018) dimension-based account of precedential constraint with 'complete' dimensions. I will then go on to present my final main contribution, in the form of an alternative to Rigoni's and Horty's dimension-based accounts of precedential constraint. The alternative is motivated by the observation (also made by BenchCapon (1999)) that in practice it is often hard to specify for some or even all value assignments to a dimension in a case whether it is for or against the case's outcome. Accordingly, I will propose a model in which all that needs to be specified is which change in value favours one outcome more and the other outcome less. Finally, I discuss how the various models of precedential constraint discussed in this paper could be applied in the HYPO and CATO systems.

This paper is organised as follows. After presenting the formal preliminaries in Sect. 2, I will in Sect. 3 define the factor-based syntactic criterion for determining forced outcomes. In Sect. 4 I discuss the relation between absence of positive factors

1 This paper is an extended and revised version of Prakken (2019). 
and presence of negative factors. In Sect. 5 I first adapt the syntactic criterion for determining forced outcomes to Horty's (2019) dimension-based result model. I then critically analyse Horty's and Rigoni's dimension-based reason models of precedential constraint and present my alternative. In Sect. 6 I address combined reasoning with factors and dimensions and in Sect. 7 I discuss the HYPO and CATO systems in light of this paper's analyses and results, after which I conclude in Sect. 8.

\section{Formal preliminaries}

In this section I review the previous work on which this paper builds.

\subsection{Citing and distinguishing cases in HYPO and CATO}

All formal work discussed in this paper is inspired by the ways cases can be cited and distinguished in the HYPO and CATO systems. Therefore I now briefly summarise these ways. HYPO and CATO generate disputes between a plaintiff and a defendant of a legal claim concerning misuse of a trade secret. Each move conforms to certain rules for analogising and distinguishing precedents given that cases contain sets of factors for and against a decision (in CATO) or dimension-value pairs (HYPO), plus the decision. A precedent is citable for side $s$ if it has the decision wished by $s$ and (in CATO) shares at least one pro-s factor with the current case or (in HYPO) the current case is on at least one dimension at least as strong for $s$ as the precedent. A citation can be countered by a counterexample, that is, by producing a citable precedent that has the opposite outcome. A citation may also be countered by distinguishing the cited precedent from the current case. In CATO this happens by indicating a factor in the current case that is absent in the cited precedent and that supports the opposite outcome, or a factor in the precedent that is missing in the current case and that supports the outcome of the cited case. In HYPO can happen by pointing at a dimension that more strongly favours the decision in the precedent than in the current case. Another way in HYPO to distinguish a citation is in terms of dimensions 'generally favouring a particular side', that is, of which all values favour the same side but to different degrees. The absence or presence of values for such dimensions allow distinguishing moves similar to those for missing or new factors, as will be discussed further in Sect. 7 .

\subsection{Notation}

I first recall some notions concerning factors and cases often used in AI \& law (e.g. by Horty (2011), Horty and Bench-Capon (2012), Rigoni (2018) and Horty (2019)), although with some differences in notation. Let $o$ and $o^{\prime}$ be two outcomes and Pro and Con be two disjoint sets of propositions called, respectively, the pro- and con factors, i.e., the factors favouring, respectively, outcome $o$ and $o^{\prime}$. The variable $s$ (for 'side') ranges over $\left\{o, o^{\prime}\right\}$ and $\bar{s}$ denotes $o^{\prime}$ if $s=o$ while it denotes $o$ if $s=o^{\prime}$. We say that a set $F \subseteq$ Pro $\cup$ Con favours side $s$ (or $F$ is pro $s$ ) if $s=o$ and $F \subseteq$ Pro or 
$s=o^{\prime}$ and $F \subseteq C o n$. For any set $F$ of factors the set $F^{s} \subseteq F$ consists of all factors in $F$ that favour side $s$. A fact situation is any subset of Pro $\cup$ Con.

The notion of a (factor-based) case can be defined in two ways. If all factors of a case $c$ are supposed to be relevant to its outcome (as in Horty's (2011) result model of precedential constraint), then it can be represented as a triple $(\operatorname{pro}(c), \operatorname{con}(c)$, outcome $(c)) \quad$ where outcome $(c) \in\left\{o, o^{\prime}\right\}$. Moreover, if outcome $(c)=o$ then $\operatorname{pro}(c) \subseteq$ Pro and $\operatorname{con}(c) \subseteq$ Con and if outcome $(c)=o^{\prime}$ then $\operatorname{pro}(c) \subseteq$ Con and $\operatorname{con}(c) \subseteq$ Pro. If, by contrast, a subset of the set of factors favouring a case's outcome can be sufficient for its outcome (as in Horty's (2011) reason model of precedential constraint), then a case can be represented as a triple $(\operatorname{ppro}(c) \cup \operatorname{con}(c), \operatorname{pro}(c)$, outcome $(c))$, where $\operatorname{pro}(c) \subseteq \operatorname{ppro}(c)$ and where the above constraints on $\operatorname{pro}(c)$ also hold for $\operatorname{ppro}(c)$ (the factors 'potentially pro' c's outcome) and the other conventions and constraints are as above. Horty calls pro $(c)$ the rule of the case. It consists of those pro-decision factors that according to the decision maker are jointly sufficient to outweigh all the con-decision factors in the case. Rigoni (2015) modifies Horty's reason model to allow for multiple rules in a case, but I will for simplicity not discuss this modification here.

Given all this, a (factor-based) case base $C B$ is a set of factor-based cases. Below I assume it clear from the context whether cases are represented for the result model or for the reason model.

\subsection{Horty's factor-based result model of precedential constraint}

I next summarise Horty's (2011) factor-based result model of precedential constraint.

Definition 1 (Preference relation on fact situations) Let $X$ and $Y$ be two fact situations. Then $X \leq_{s} Y$ iff $X^{s} \subseteq Y^{s}$ and $Y^{\bar{s}} \subseteq X^{\bar{s}}$.

$X<_{s} Y$ is defined as usual as $X \leq_{s} Y$ and $Y \not_{s} X$. This definition says that $Y$ is at least as good for $s$ as $X$ iff $Y$ contains at least all pro-s factors that $X$ contains and $Y$ contains no pro- $\bar{s}$ factors that are not in $X$.

Definition 2 (Precedential constraint with factors: result model) Let $C S$ be a case base and $F$ a fact situation. Then, given $C B$, deciding $F$ for $s$ is forced iff there exists a case $c=(X, Y, s)$ in $C B$ such that $X \cup Y \leq_{s} F$.

Horty thus models a fortiori reasoning in that an outcome in a focus case is forced if a precedent with the same outcome exists such that all their differences make the focus case at least as strong for their outcome as the precedent.

As the first running example I use a small part of the US trade secrets domain of the HYPO and CATO systems. I assume the following six factors from CATO along with whether they favour the outcome 'misuse of trade secrets' ( $\pi$ for 'the plaintiff wins') or 'no misuse of trade secrets' ( $\delta$ for 'the defendant wins'): the defendant had obtained the secret by deceiving the plaintiff $\left(\pi_{1}\right)$ or by bribing 
an employee of the plaintiff $\left(\pi_{2}\right)$, the plaintiff had taken security measures to keep the secret $\left(\pi_{3}\right)$, the information is obtainable elsewhere $\left(\delta_{1}\right)$, the product is reverse-engineerable $\left(\delta_{2}\right)$ and the plaintiff had voluntarily disclosed the secret to outsiders $\left(\delta_{3}\right)$. We assume the following precedents:

$c_{1}(\pi)$ : deceived $_{\pi 1}$, measure $_{\pi 3}$, obtainable-elsewhere $_{\delta 1}$, disclosed $_{\delta 3}$

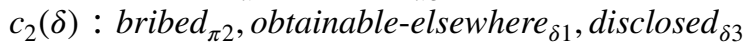

Clearly, given this case base, deciding a fact situation $F$ for $\pi$ is forced iff it has at least the $\pi$-factors $\left\{\pi_{1}, \pi_{3}\right\}$ and at most the $\delta$-factors $\left\{\delta_{1}, \delta_{3}\right\}$ (by precedent $c_{1}$ ), since then we have $\left\{\pi_{1}, \pi_{3}\right\} \subseteq F^{\pi}$ and $F^{\delta} \subseteq\left\{\delta_{1}, \delta_{3}\right\}$. Likewise, deciding a fact situation for $\delta$ is forced iff it has at least the $\delta$-factors $\left\{\delta_{1}, \delta_{3}\right\}$ and at most the $\pi$-factor $\left\{\pi_{2}\right\}$ (by precedent $c_{2}$ ).

Consider next the following fact situation:

$$
F_{1}: \text { bribed }_{\pi 2}, \text { measures }_{\pi 3}, \text { reverse-eng }_{\delta 2}, \text { disclosed }_{\delta 3}
$$

Comparing $F_{1}$ with precedent $c_{1}$ we must check whether $\left\{\pi_{1}, \pi_{3}, \delta_{1}, \delta_{3}\right\} \leq_{\pi}\left\{\pi_{2}, \pi_{3}, \delta_{2}, \delta_{3}\right\}$. This is not the case, for two reasons. We have $\left\{\pi_{1}, \pi_{3}\right\} \nsubseteq F_{1}^{\pi}=\left\{\pi_{2}, \pi_{3}\right\}$ and we have $F_{1}^{\delta}=\left\{\delta_{2}, \delta_{3}\right\} \nsubseteq\left\{\delta_{1}, \delta_{3}\right\}$. Next, comparing with precedent $c_{2}$ we must check whether $\left\{\pi_{2}, \delta_{1}, \delta_{3}\right\} \leq_{\delta}\left\{\pi_{2}, \pi_{3}, \delta_{2}, \delta_{3}\right\}$. This is also not the case for two reasons. We have $\left\{\delta_{1}, \delta_{3}\right\} \nsubseteq F_{1}^{\delta}=\left\{\delta_{2}, \delta_{3}\right\}$ and we have $F_{1}^{\pi}=\left\{\pi_{2}, \pi_{3}\right\} \nsubseteq\left\{\pi_{2}\right\}$. So neither deciding $F_{1}$ for $\pi$ nor deciding $F_{1}$ for $\delta$ is forced.

\subsection{Horty's factor-based reason model of precedential constraint}

I finally summarise Horty's (2011) factor-based reason model of precedential constraint. The following definition says that a case decision expresses a preference for any pro-decision set containing at least the pro-decision factors of the case over any con-decision set containing at most the con-decision factors of the case. This allows a fortiori reasoning from a precedent adding pro-decision factors and/or deleting con-decision factors.

Definition 3 (Preferences from cases with factors) Let (ppro(c) $\cup \operatorname{con}(c), \operatorname{pro}(c), s)$ be a case, $C B$ a case base and $X$ and $Y$ sets favouring $s$ and $\bar{s}$, respectively. Then

1. $Y<{ }_{c} X$ iff $Y \subseteq \operatorname{con}(c)$ and $X \supseteq \operatorname{pro}(c)$;

2. $Y<_{C B} X$ iff $Y<_{c} X$ for some $c \in C B$.

Definition 4 ((In)consistent case bases) Let $C$ be a case base with $<_{C B}$ the derived preference relation. Then $C B$ is inconsistent if and only if there are factor sets $X$ and $Y$ such that $X<_{C B} Y$ and $Y<_{C B} X$. And $C B$ is consistent if and only if it is not inconsistent. 
The final definition says that deciding a case for a particular outcome is forced if that is the only way to keep the updated case base consistent. ${ }^{2}$

Definition 5 (Precedential constraint with factors: reason model) Let $C B$ be a case base and $F$ a fact situation. Then, given $C B$, deciding $F$ for $s$ is forced iff for all cases $c=\left(F, R^{\prime}\right.$, outcome $\left.(c)\right)$ such that $C B \cup\{c\}$ is consistent it holds that outcome $(c)=s$.

Horty adds to this the constraint that the court should select a rule $R$ such that $C B \cup\{(F, R, s)\}$ is consistent whenever $C B$ is consistent. Note that if the $C B$ is inconsistent, then Definition 5 implies that both deciding for $s$ and deciding for $\bar{s}$ is forced. Horty proves that for consistent cases bases his result and reason model are equivalent on the assumption that $\operatorname{pro}(c)=\operatorname{ppro}(c)$ for all cases $c$.

Suppose that in our running example the cases are modified to the effect

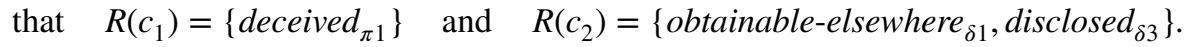
Then for $c_{2}$ the analysis is as in the result model but for $c_{1}$ it is different. Now deciding a fact situation $F$ for $\pi$ is forced iff it has at least the $\pi$-factor deceived $_{\pi 1}$ and at most the $\delta$-factors $\left\{\right.$ obtainable-elsewhere $_{\delta 1}$, disclosed $\left._{\delta 3}\right\}$, since $\left\{\right.$ obtainable-elsewhere $_{\delta 1}$, $_{\text {isclosed }}$ dis $\left._{\delta}\right\}<_{c 1}$ deceived $\left._{\pi 1}\right\}$, so for any $F$ such

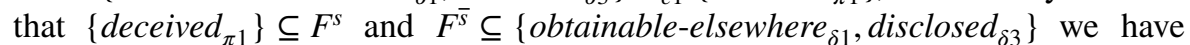
that $F^{\bar{s}}<_{c} F^{s}$, so deciding $F$ for $\bar{s}$ yields $F^{s}<{ }_{c} F^{\bar{s}}$ and so makes the case base inconsistent.

\section{Relevant differences in precedential constraint with factors}

In this section I formally define a notion of the relevant differences between a precedent and a focus case (i.e., a case to be decided). I then prove that it yields a simple syntactic criterion for checking whether some outcome in the focus case is forced. The relevant differences between a precedent and the focus case are those differences that are a reason not to decide the focus case as the precedent. These are the situations in which a precedent can be distinguished in a HYPO/CATO-style approach (Ashley 1989; Aleven and Ashley 1991), namely, when the new case lacks some factors pro its outcome that are in the precedent or has new factors con its outcome that are not in the precedent. Ashley (1990, Section 9.1) also presents a definition of relevant differences between cases, although not for the purpose of characterising precedential constraint. I will discuss his definition in Sect. 7.

In defining the relevant differences between two cases, it is relevant whether the two cases have the same outcome or different outcomes. If they have the same outcome, then a factor in the precedent that lacks in the focus case is only relevant if it is pro that outcome; otherwise its absence in the focus case only makes the focus case stronger than the precedent. Likewise, an additional factor in the focus case is only relevant if it is con the outcome. If the focus case and the precedent

\footnotetext{
2 This definition corrects the one in Prakken (2019), which differs from Horty's for inconsistent case bases.
} 
have opposite outcomes, then this becomes different. If the precedent has an additional factor that is pro the outcome in the precedent (so con the outcome in the focus case), then its missing in the focus case makes the focus case stronger than the other case, so it is a relevant difference. However, if the additional factor in the precedent is con the outcome in that case, then its missing in the focus case as (as a factor pro its outcome) makes the focus case weaker compared to the precedent. So this is not a relevant difference. On the other hand, if the focus case has an additional factor pro its outcome, then its addition to the precedent (as a factor con its outcome) might have changed its outcome, so this is a relevant difference between the cases. Finally, if the additional factor in the focus case is con its outcome, then adding it to the precedent (as a factor pro its outcome) strengthens the precedent, so this is not a relevant difference between the cases.

Definition 6 (Differences between cases with factors) Let $c$ and $f$ be two cases. The set $D(c, f)$ of differences between $c$ and $f$ is defined as follows.

1. If outcome $(c)=$ outcome $(f)$ then $D(c, f)=\operatorname{pro}(c) \backslash \operatorname{pro}(f) \cup \operatorname{con}(f) \backslash \operatorname{con}(c)$.

2. If outcome $(c) \neq$ outcome $(f)$ then $D(c, f)=\operatorname{pro}(f) \backslash \operatorname{con}(c) \cup \operatorname{pro}(c) \backslash \operatorname{con}(f)$.

Consider again our running example and consider first any focus case $f$ with outcome $\pi$ and with a fact situation that has at least the $\pi$-factors deceived $_{\pi 1}$, $_{\text {easures }}$ en $\left._{3}\right\}$ and at most the $\delta$-factors \{rbtainable-elsewhere $_{\delta 1}$, disclosed $\left._{\delta 3}\right\}$. Then $D(c, f)=\emptyset$. Likewise with any focus case $f$ with outcome $\delta$ and with a fact situation that has at least the $\delta$-factors

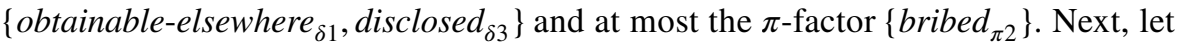
$f$ be a focus case with fact situation $F_{1}$ and outcome $\pi$. We have

$$
\begin{aligned}
& D\left(c_{1}, f\right)=\left\{\text { deceived }_{\pi 1}, \text { reverse-eng }_{\delta 2}\right\} \\
& D\left(c_{2}, f\right)=\left\{\text { measures }_{\pi 3}, \text { obtainable-elsewhere }_{\delta 1}\right\}
\end{aligned}
$$

I next show for the reason model that for consistent case bases the above definition of relevant differences can be used to check whether a specific outcome of a new case is forced. Since the result model is the special case of the reason model where $R=F^{s}$, this result also holds for the result model.

Proposition 1 Let $C B$ be a consistent case base $C B$ and $F$ a fact situation. Then deciding $F$ for $s$ is forced according to the reason model given $C B$ iff there exists a case $c$ with outcome $s$ in $C B$ such that $D(c, f)=\emptyset$, where $f=\left(F, F^{s}, s\right)$.

Proof Assume first that deciding $F$ for $s$ is forced. Then every case $f^{\prime}=\left(F^{\bar{s}} \cup F^{s}, R^{\prime}, \bar{s}\right)$ is inconsistent with the case base. Let without loss of generality $R^{\prime}=F^{\bar{s}}$. Then since $C B$ is consistent, by Observation 1 of Horty (2019) there exists a case $c=\left(X \cup Y, R^{\prime \prime}, s\right) \in C B$ such that $R^{\prime \prime}<_{f^{\prime}} F^{\bar{s}}$ and $F^{\bar{s}}<_{c} R^{\prime \prime}$. The former priority entails that $R^{\prime \prime} \subseteq F s$. But then $\operatorname{pro}(c) \subseteq \operatorname{pro}(f)$, so (1) pro $(c) \backslash \operatorname{pro}(f)=\emptyset$. The latter priority entails that $F^{\bar{s}} \subseteq Y$. But then $(2) \operatorname{con}(f) \subseteq \operatorname{con}(c)$ so $(2) \operatorname{con}(f) \backslash \operatorname{con}(c)=\emptyset$. Then observe that (1) and (2) together entail that $D(c, f)=\emptyset$. 
Assume next that there exists a $c \in C B$ with outcome $s$ and such that $D(c, f)=\emptyset$. Then we have $\operatorname{con}(c)<_{c} \operatorname{pro}(c)$ and we have $\operatorname{pro}(c) \subseteq \operatorname{pro}(f)$ and $\operatorname{con}(f) \subseteq \operatorname{con}(c)$. But then we also have $\operatorname{con}(f)<_{c} \operatorname{pro}(f)$, so for every $R \subseteq \operatorname{con}(f)$ we have $R<_{c} \operatorname{pro}(f)$ and so $R<_{c}$ ppro $(f)$. Any rule for deciding the facts of $f$ for $\bar{s}$ requires adding a case $c^{\prime}=(F, R, \bar{s})$ to $C B$ but then ppro $(f)<_{c} R$ can be derived from $C B$, so $C B$ is inconsistent.

For consistent case bases this proposition yields a simple syntactic criterion for determining whether a decision is forced. In this case it allows us to rewrite Definition 5 by writing that deciding $F$ for $s$ is forced iff there exists a case $c=(\operatorname{ppro}(c) \cup \operatorname{con}(c), \operatorname{pro}(c), s)$ in $C B$ such that $\operatorname{pro}(c) \subseteq F^{s}$ and $F^{\bar{s}} \subseteq \operatorname{con}(c)$. The latter can in turn with Definition 1 in the style of Definition 2 be rewritten as: ... such that $\operatorname{pro}(c) \cup \operatorname{con}(c) \leq_{s} F$.

Proposition 1 does not in general hold for inconsistent case bases, since for such case bases Definition 5 implies that every decision is forced, including decisions that have relevance differences with the focus case. However, it does hold for inconsistent knowledge bases for the result model.

Proposition 2 Let $C B$ be a case base $C B$ and $F$ a focus case. Then deciding $F$ for $s$ is forced according to the result model given $C B$ iff there exists a case $c$ with outcome $s$ in $C B$ such that $D(c, f)=\emptyset$, where $f=\left(F, F^{s}, s\right)$.

Proof Assume first that deciding $F$ for $s$ is forced. Then there exists a case $c=(X, Y, s)$ in $C B$ such that $X \cup Y \leq_{s} F$. But then $X \subseteq F^{s}$ and $F^{\bar{s}} \subseteq Y$, so $D(c, f)=\emptyset$

Assume next that there exists a case $c$ with outcome $s$ in $C B$ such that $D(c, f)=\emptyset$. Then $X \subseteq F^{s}$ and $F^{\bar{s}} \subseteq Y$, so $X \cup Y \leq_{s} F$.

Another result is that for any two cases with opposite outcomes that both have differences with the focus case, their sets of differences with the focus case are mutually incomparable (as with $c_{1}$ and $c_{2}$ in our running example).

Proposition 3 Let CB be a case base, f a focus case and c and $c^{\prime}$ two cases with opposite outcomes and with non-empty sets of differences with $f$. Then $D(c, f) \nsubseteq D\left(c^{\prime}, f\right)$ and $D\left(c^{\prime}, f\right) \nsubseteq D(c, f)$.

Proof Suppose $c$ and $f$ have the same outcome and suppose that $\varphi \in D(c, f)$. Assume first $\varphi \in \operatorname{pro}(c) \backslash \operatorname{pro}(f)$. Then $\varphi \notin \operatorname{pro}(f)$, so $\varphi \notin \operatorname{pro}(f) \backslash \operatorname{con}\left(c^{\prime}\right)$, so $\varphi \notin D\left(c^{\prime}, f\right)$.

Assume next $\varphi \in \operatorname{con}(f) \backslash \operatorname{con}(c)$. Then $\varphi \in \operatorname{con}(f)$ so $\varphi \notin \operatorname{pro}\left(c^{\prime}\right) \backslash \operatorname{con}(f)$, so $\varphi \notin D\left(c^{\prime}, f\right)$.

Suppose now that $c$ and $f$ have different outcomes and suppose that $\varphi \in D(c, f)$. Assume first $\varphi \in \operatorname{pro}(f) \backslash \operatorname{con}(c)$. Then $\varphi \in \operatorname{pro}(f)$, so $\varphi \notin \operatorname{pro}\left(c^{\prime}\right) \backslash \operatorname{pro}(f)$, so $\varphi \notin D\left(c^{\prime}, f\right)$.

Assume next $\varphi \in \operatorname{pro}(c) \backslash \operatorname{con}(f)$. Then $\varphi \notin \operatorname{con}(f)$, so $\varphi \notin \operatorname{con}(f) \backslash \operatorname{con}\left(c^{\prime}\right)$, so $\varphi \notin D\left(c^{\prime}, f\right)$. 


\section{Positive and negative factors}

An interesting question is whether the fact that a factor favouring one side does not apply in a case favours the other side. In other words, should a closed-world assumption be applied that when a factor is absent from a case, then the negated factor favouring the other side should be included in the set of factors? Horty (2011, pp. 26-27) suggests that it may be sensible to decide for each factor separately whether such a closed-world assumption should be applied to it. Horty refers to Aleven (1997, pp. 239-247) as support for his suggestion, who for each CATO factor separately lists whether the closed-world assumption applies to it, for the purpose of identifying whether a distinction on this factor can be 'emphasised' in CATO by pointing at a more abstract absent factor favouring the same side. Rissland and Ashley (2002, pp.67-69) argue that in applications like HYPO and CATO a negated factor favouring the other side can only be included in the set of factors if a case can be found where judges had said that the absence of a factor favouring one side favours the other side. This can be been seen as an application of the idea to consider each factor separately instead of applying a general closed-world assumption to the set of all factors.

It is interesting to investigate to which extent the possible differences in approaches matter within the result and reason models of precedential constraint. It turns out that they only matter in the reason model. Recall that Pro and Con are the factors favouring, respectively, outcomes $o$ and $o^{\prime}$. I now assume that Pro $\cup$ Con does not contain both a factor $x$ and its negation $\neg x$; this assumption prevents that a negated factor is introduced by the closed-world assumption is already present in the factor in non-negated form. Let the completion of Pro $\cup$ Con with respect to $C \subseteq$ Pro $\cup$ Con be defined as the set $P r o\urcorner \cup C o n\urcorner$, where

$$
\text { Pro }\urcorner=\operatorname{Pro} \cup\{\neg x \mid x \in C \cap \operatorname{Con}\} \text { and } \operatorname{Con}\urcorner=\operatorname{Con} \cup\{\neg x \mid x \in C \cap \text { Pro }\} .
$$

The relativity of a completion to a given set $C$ of factors is meant to accommodate Horty's suggestion to decide for each factor separately whether the closed-world assumption applies to it. Then in the result model the completion $c\urcorner$ of a factor-based case $c=(X, Y, o)$ given the completion of Pro $\cup$ Con with respect to $C$ is defined as:

$$
c\urcorner=(X \cup\{\neg x \in P r o\urcorner \mid x \in C o n \text { and } x \notin Y\}, Y \cup\{\neg x \in C o n\urcorner \mid x \in \text { Pro and } x \notin X\}, o) \text {. }
$$

Likewise, the completion of a fact situation $F$ where $X=F^{s}$ and $Y=F^{\bar{s}}$ given the completion of Pro $\cup$ Con with respect to $C$ is defined as:

$$
F\urcorner=(X \cup\{\neg x \in P r o\urcorner \mid x \in C o n \text { and } x \notin Y\}, Y \cup\{\neg x \in C o n\urcorner \mid x \in \text { Pro and } x \notin X\}, o) \text {. }
$$

Then the completion $C B\urcorner$ of a factor-based case base $C B$ given the completion of Pro $\cup$ Con with respect to $C$ is the case base obtained by replacing each $c \in C B$ with $c\urcorner$ given the completion.

The following result on sets of relevant differences can be proven. 
Proposition 4 Let $c$ and $f$ be two factor-based cases in the result model with the same outcome s. Consider any completion $\mathcal{C}$ of Pro $\cup$ Con and let $c\urcorner$ and $f\urcorner$ be the completions of $c$ and $f$ given $\mathcal{C}$. Then $D(c, f)=\emptyset$ if and only if $D(c\urcorner, f\urcorner)=\emptyset$.

Proof Let without loss of generality $s=o$. For the only-if part, assume that $D(c, f)=\emptyset$. Then (1) $\operatorname{pro}(c) \subseteq \operatorname{pro}(f)$ and (2) $\operatorname{con}(f) \subseteq \operatorname{con}(c)$. Furthermore, let $\mathcal{C}$ be a completion with respect to set $C$. Then we have (3) $\quad \operatorname{pro}(c\urcorner)=\operatorname{pro}(c) \cup\{\neg x \mid x \in \operatorname{Con} \cap C \backslash \operatorname{con}(c)\}, \quad$ and $\quad$ (4) $\operatorname{pro}(f\urcorner)=\operatorname{pro}(f) \cup\{\neg x \mid x \in \operatorname{Con} \cap C \backslash \operatorname{con}(f)\} . \quad$ Moreover, the following set-theoretic equivalence holds: $\operatorname{con}(f) \subseteq \operatorname{con}(c)$ if and only if $\operatorname{Con} \backslash \operatorname{con}(c) \subseteq \operatorname{Con} \backslash \operatorname{con}(f)$. The right-hand-side of this equivalence implies Con $\cap C \backslash \operatorname{con}(c) \subseteq \operatorname{Con} \cap C \backslash \operatorname{con}(f)$. Then given (1) and (2) we have by (3) and (4) that $\operatorname{pro}(c\urcorner) \subseteq \operatorname{pro}(f\urcorner)$.

To prove that $\operatorname{con}(f\urcorner) \subseteq \operatorname{con}(c\urcorner)$, note that we also have $\operatorname{con}(c\urcorner)=\operatorname{con}(c) \cup\{\neg x \mid x \in \operatorname{Pro} \cap C \backslash \operatorname{pro}(c)\}, \quad$ and $\operatorname{con}(f\urcorner)=\operatorname{con}(f) \cup\{\neg x \mid x \in \operatorname{Pro} \cap C \backslash \operatorname{pro}(f)\}$. Then the proof is as above. So $D(c\urcorner, f\urcorner)=\emptyset$.

For the only-if part, assume that $D(c\urcorner, f\urcorner)=\emptyset$. Then $\operatorname{pro}(c\urcorner) \subseteq \operatorname{pro}(f\urcorner)$ and $\operatorname{con}(f\urcorner) \subseteq \operatorname{con}(c\urcorner)$. Assume for contradiction that $\operatorname{pro}(c) \nsubseteq \operatorname{pro}(f)$. Then there exists a $p \in \operatorname{pro}(c)$ such that $p \notin \operatorname{pro}(f)$. Then $\neg p \notin \operatorname{con}(c\urcorner)$ but $\neg p \in \operatorname{con}(f\urcorner)$. But then $\operatorname{con}(f\urcorner) \nsubseteq \operatorname{con}(c\urcorner)$. Contradiction, so $\operatorname{pro}(c) \subseteq \operatorname{pro}(f)$.

Assume next for contradiction that $\operatorname{con}(f) \nsubseteq \operatorname{con}(c)$. Then there exists a $p \in \operatorname{con}(f)$ such that $p \notin \operatorname{con}(c)$. Then $\neg p \notin \operatorname{pro}(f\urcorner)$ but $\neg p \in \operatorname{pro}(c\urcorner)$. But then $\operatorname{pro}(c\urcorner) \nsubseteq \operatorname{pro}(f\urcorner)$. Contradiction, so $\operatorname{con}(f) \subseteq \operatorname{con}(c)$.

Corollary 1 Let $C B$ be a factor-based case base with its completed counterpart $C B\urcorner$ and $F$ a fact situation with completion $F\urcorner$. Then deciding $F$ for $s$ is forced given $C B$ according to Definition 2 if and only if deciding $F\urcorner$ for $s$ is forced given $C B\urcorner$ according to Definition 2.

For the reason model the analysis is different. First, in the reason model the completion $c\urcorner$ of a factor-based case $c=\left(X \cup Y, X^{\prime}, o\right)$ given the completion of Pro $\cup$ Con with respect to $C$ is defined as:

$$
\left.c\urcorner=(X \cup\{\neg x \in P r o\urcorner \mid x \in C o n \text { and } x \notin Y\} \cup(Y \cup\{\neg x \in C o n\urcorner \mid x \in \text { Pro and } x \notin X\}), X^{\prime}, o\right) .
$$

In other words, a completion of case $c$ says that the pro-outcome factors in the rule suffice to outweigh the union of the original con-outcome factors and the absent prooutcome factors that are subject to the closed-world assumption. With this definition, the only-if part of Proposition 4 does not hold for the reason model, since then we do not always have equations (5) and (6) in the proof. For a counterexample, let Pro $=\left\{p_{1}, p_{2}\right\}$, Con $=\emptyset, c=\left(\left\{p_{1}, p_{2}\right\},\left\{p_{1}\right\}, s\right)$ and $f=\left(\left\{p_{1}\right\}, \emptyset, s\right)$ and we complete with respect to Pro. We have that $c\urcorner=c$ and $f\urcorner=\left(\left\{p_{1}\right\},\left\{\neg p_{2}\right\}, s\right.$. Then $D(c, f)=\emptyset$ while $D(c\urcorner, f\urcorner)=\left\{\neg p_{2}\right\}$. The point is that the negation of the pro- $o$ factor $p_{2}$, which is not in the rule of $c$, becomes a new con- $o$ factor in $f$ after completion. In the result model this cannot happen, since then the absence of $p_{2}$ in $f$ is a relevant difference 
between $c$ and $f$, so $D(c, f) \neq \emptyset$. This also yields a counterexample to the only-if part of Corollary 1.

However, the if-parts of the above results do hold for the reason model.

Proposition 5 Let $c$ and $f$ be two factor-based cases in the reason model with the same outcome s. Consider any completion $\mathcal{C}$ of Pro $\cup$ Con and let $c\urcorner$ and $f\urcorner$ be the completions of $c$ and $f$ given $\mathcal{C}$. Then $D(c, f)=\emptyset$ if $D(c\urcorner, f\urcorner)=\emptyset$.

Proof Assume that $D(c\urcorner, f\urcorner)=\emptyset$. Then $\operatorname{pro}(c\urcorner) \subseteq \operatorname{pro}(f\urcorner)$ and $\operatorname{con}(f\urcorner) \subseteq \operatorname{con}(c\urcorner)$. Since the rule of a case does not change in a completion, it is immediate that $\operatorname{pro}(c) \subseteq \operatorname{pro}(f)$.

Assume next for contradiction that $\operatorname{con}(f) \nsubseteq \operatorname{con}(c)$. Then there exists a $p \in \operatorname{con}(f)$ such that $p \notin \operatorname{con}(c)$. But $p \in \operatorname{con}(f)$ implies $p \in \operatorname{con}(f\urcorner)$ since Pro $\cup$ Con does not contain both a factor $x$ and its negation $\neg x$. But then $d \in \operatorname{con}(c)$. Contradiction, so $\operatorname{con}(f) \subseteq \operatorname{con}(c)$.

These results mean that the result model cannot discriminate between two answers to the question what it means to say that a factor is absent in a case. In, for example, the trade-secrets domain the absence of the factor disclosed could either mean that there were no disclosures to outsiders or that there is no information about whether there were such disclosures. The result model can, unlike the reason model, not distinguish between these two meanings.

\section{Adapting the approach to dimensions}

In this section I discuss various ways in which the above models can be and have been adapted to dimensions. I first adapt the above factor-based definition of relevant differences to Horty's dimension-based result model (Horty 2019). Then I will not do the same for Horty's dimension-based reason models, for two reasons. First, as Horty himself shows, his (2019) dimension-based reason model collapses into his result model, which arguably fails to capture the distinction between ratio decidendi and obiter dicta familiar from common-law jurisdictions. Although Horty (2020) presents a revision of his reason model that does not collapse into his result model, it shares a feature with his original model (which is even more strongly present in Rigoni's 2018 model) which raises some concerns about practical applicability. For these reasons I will first formally reconstruct Rigoni's (2018) model for so-called complete dimensions and then present an alternative which, although less expressive than Horty's and Rigoni's reaosn models, is arguably easier to apply in practice.

\subsection{Relevant differences in Horty's dimension-based result model}

I adopt from Horty (2019) the following technical ideas (again with some notational differences). A dimension is a tuple $d=\left(V, \leq_{o}, \leq_{o^{\prime}}\right)$ where $V$ is a set (of values) and $\leq_{o}$ and $\leq_{o^{\prime}}$ two partial orders on $V$ such that $v \leq_{o} v^{\prime}$ iff $v^{\prime} \leq_{o^{\prime}} v$. A value 
assignment is a pair $(d, v)$. The functional notation $v(d)=x$ denotes the value $x$ of dimension $d$. Then a (dimension-based) case is a pair $c=(F$, outcome $(c))$ such that $D$ is a set of dimensions, $F$ is a set of value assignments to all dimensions in $D$ and outcome $(c) \in\left\{o, o^{\prime}\right\}$. Then a (dimension-based) case base is as before a set of cases, but now explicitly assumed to be relative to a set $D$ of dimensions in that all cases assign values to a dimension $d$ iff $d \in D$. Likewise, a (dimension-based) fact situation is now an assignment of values to all dimensions in $D$. As for notation, $v(d, c)$ denotes the value of dimension $d$ in case or fact situation $c$. Finally, $v \geq_{s} v^{\prime}$ is the same as $v^{\prime} \leq_{s} v$. The assumption that all dimensions have a value in each case is, of course, a genuine limitation, but since it is also made by Horty (2019), I leave a study of models without this assumption to future research.

In HYPO (Rissland and Ashley 1987; Ashley 1990), two of CATO's factors from our running example are actually dimensions. Security-Measures-Adopted has a linearly ordered range, below listed in simplified form (where later items increasingly favour the plaintiff so decreasingly favour the defendant):

Minimal-Measures, Access-To-Premises-Controlled, Entry-By-Visitors-

Restricted,

Restrictions-On-Entry-By-Employees

(For simplicity I will below assume that each case contains exactly one security measure; generalisation to multiple measures is straightforward by defining the orderings between sets of measures.) Moreover, disclosed has a range from 1 to some high number, where higher numbers increasingly favour the defendant so decreasingly favour the plaintiff. For the remaining four factors I assume that they have two values 0 and 1 , where presence (absence) of a factor means that its value is $1(0)$ and where for the pro-plaintiff factors we have $0<_{\pi} 1\left(\right.$ so $\left.1<_{\delta} 0\right)$ and for the pro-defendant factors we have $0<_{\delta} 1$ ( so $1<_{\pi} 0$ ).

Accordingly, I change the running example as follows.

$c_{1}(\pi):$ deceived $_{\pi 1}$, measures $=$ Entry-By-Visitors-Restricted, obtainable-elsewhere $_{\delta 1}$, disclosed $=10$

$c_{2}(\delta):$ bribed $_{\pi 2}$, measures $=$ Minimal, obtainable-elsewhere ${ }_{\delta 1}$, disclosed $=5$

$F_{1}: \quad$ bribed $_{\pi 2}$, measures $=$ Access-To-Premises-Controlled, reverse-eng ${ }_{\delta 2}$, disclosed $=20$

In Horty's result model a decision in a fact situation is forced iff there exists a precedent $c$ for that decision such that on each dimension the fact situation is at least as favourable for that decision as the precedent. Horty formalises this idea with the help of the following preference relation between sets of value assignments.

Definition 7 (Preference relation on dimensional fact situations) Let $F$ and $F^{\prime}$ be two fact situations with the same set of dimensions. Then $F \leq_{s} F^{\prime}$ iff for all $(d, v) \in F$ and all $\left(d, v^{\prime}\right) \in F^{\prime}$ it holds that $v \leq_{s} v^{\prime}$.

In our running example we have for any fact situation $F^{\prime}$ that $F\left(c_{1}\right) \leq_{\pi} F^{\prime}$ iff $F^{\prime}$ has $\pi_{1}$ but not $\delta_{3}$ and $v$ (measures, $\left.F^{\prime}\right) \geq_{\pi}$ Entry-By-Visitors-Restricted and 
$v\left(\right.$ disclosed,$\left.F^{\prime}\right) \geq_{\pi} 20$ (so $\leq 20$ ). Likewise, $F\left(c_{2}\right) \leq_{\delta} F^{\prime}$ iff $F^{\prime}$ has $\delta_{1}$ but not $\pi_{1}$ and $v\left(\right.$ measures,$\left.F^{\prime}\right)=$ Minimal and $v\left(\right.$ disclosed,$\left.F^{\prime}\right) \geq_{\delta} 10$ (so $\left.\geq 10\right)$.

Then adapting Horty's factor-based result model to dimensions is straightforward.

Definition 8 (Precedential constraint with dimensions: result model) Let $C S$ be a case base and and $F$ a fact situation given a set $D$ of dimensions. Then, given $C B$, deciding $F$ for $s$ is forced iff there exists a case $c=\left(F^{\prime}, s\right)$ in $C B$ such that $F^{\prime} \leq_{s} F$.

In our running example, deciding $F_{1}$ for $\pi$ is not forced, for two reasons. First, $v\left(c_{1}\right.$, deceived $)=1$ while $v\left(F_{1}\right.$, deceived $)=0$ and for deceived we have that $0<_{\pi} 1$. Second, $v\left(c_{1}\right.$, measures $)=$ Entry-By-Visitors-Restricted while $v\left(F_{1}\right.$, measures $)=$ Access-ToPremises - Controlled and Access-To-Premises-Controlled $<_{\pi} \quad$ Entry-By-VisitorsRestricted. Moreover, deciding $F_{1}$ for $\delta$ is also not forced, since $v\left(c_{2}\right.$, measures $)=$ Minimal while $v\left(F_{1}\right.$, measures $)=$ Access-To-Premises-ControlledandMinimal $<_{\delta}$ Access-To-Premises-Controlled.

I next adapt Definition 6 of differences between cases to dimensions. Note that unlike in the case of factors, there is no need to indicate whether a value assignment favours a particular side in the case, since the $\leq_{s}$ ordering suffices for this purpose.

Definition 9 (Differences between cases with dimensions: result model) Let $c=(F(c)$,outcome $(c))$ and $f=(F(f)$,outcome $(f))$ be two cases. The set $D(c, f)$ of differences between $c$ and $f$ is defined as follows.

1. If outcome $(c)=$ outcome $(f)=s$ then $D(c, f)=\left\{(d, v) \in F(c) \mid v(d, c) \not \mathbf{Z}_{s} v(d, f)\right.$.

2. If outcome $(c) \neq$ outcome $(f) \quad$ whe r e outcome $(c)=s \quad$ th e $\mathrm{n}$ $D(c, f)=\left\{(d, v) \in F(c) \mid v(d, c) \Varangle_{\bar{s}} v(d, f)\right.$.

Let $c$ be a precedent and $f$ a focus case. Then clause (1) says that if the outcomes of the precedent and the focus case are the same, then any value assignment in the focus case that is not at least as favourable for the outcome as in the precedent is a relevant difference. Clause (2) says that if the outcomes are different, then any value assignment in the focus case that is not at most as favourable for the outcome of the focus case as in the precedent is a relevant difference.

In our running example, we have:

$$
\begin{aligned}
& D\left(c_{1}, f\right)=\{(\text { deceived }, 1),(\text { measures }, \text { Entry-By-Visitors-Restricted })\} \\
& D\left(c_{2}, f\right)=\{(\text { measures }, \text { Minimal })\}
\end{aligned}
$$

We have the following counterpart of Proposition 2 for Horty's dimension-based result model.

Proposition 6 Let, given a set $D$ of dimensions, $C B$ be a case base and $F$ a fact situation. Then deciding $F$ for $s$ is forced given $C B$ according to Definition 8 iff there exists a case in $C B$ with outcome such that $D(c, f)=\emptyset$, where $f=(F, s)$. 
Proof Consider first any $c=(F(c), s)$ in $C B$ such that $D(c, f)=\emptyset$. Then for all $(d, v) \in F(c)$ and all $\left(d, v^{\prime}\right) \in F(f)$ it holds that $v \leq_{s} v^{\prime}$, so $F(c) \leq_{s} F(f)$.

Suppose next $f$ is forced. Then the proof is the same the other way around.

The counterpart of Proposition 3 can be proven as a new result.

Proposition 7 Let, given a set $D$ of dimensions, $C B$ be a case base, $f$ a focus case and $c$ and $c^{\prime}$ be two cases with opposite outcomes and both with a non-empty set of differences with $f$. Then $D(c, f) \nsubseteq D\left(c^{\prime}, f\right)$ and $D\left(c^{\prime}, f\right) \nsubseteq D(c, f)$.

Proof Suppose first that $c$ and $f$ have the same outcome and suppose that $(d, v) \in D(c, f)$. Then $v(d, c) \varliminf_{s} v(d, f)$, so $v(d, c) \Varangle_{s} v(d, f)$, so $(d, v) \notin D\left(c^{\prime}, f\right)$. Suppose next that $c$ and $f$ have different outcomes and suppose that $(d, v) \in D(c, f)$. Then $v(d, c) \Varangle_{\bar{s}} v(d, f)$, so $v(d, c) \not_{s} v(d, f)$, so $(d, v) \notin D\left(c^{\prime}, f\right)$.

\subsection{A dimension-based reason model with complete rules}

I next discuss how Horty's dimension-based result model can be turned into a dimension-based reason model. There are two features on which this can be done: by 'relaxing' an individual value assignment or by leaving some assignments out from a set of value assignments. In both ways a case is a triple $(c=(F(c), R(c)$, outcome $(c))$, where $F(c)$ is as in the result model a value assignment to a given set $D$ of dimensions and where $R(c)$, the rule of the case, is a set of value assignments that in some way constrained by $F(c)$. In the first way, rule $R(c)$ consists of value assignments to each dimension in $D$ such that for each element $(d, v)$ in $R(c)$ and each element $\left(d, v^{\prime}\right)$ in $F(c)$ it holds that $v(d) \leq_{s} v^{\prime}(d)$. In other words, in this approach a rule of a case assigns to each of the case's dimensions a value that is at most as favourable for the case's outcome as its value in the case. Below I will call such a rule a complete rule.

Definition 10 (Precedential constraint with dimensions: a reason model with complete rules) Let, given a set $D$ of dimensions, $C S$ be a case base in which all cases have a complete rule and $F$ a fact situation. Then deciding $F$ for $s$ is forced iff there exists a case $c=\left(F^{\prime}, R, s\right)$ in $C B$ such that $R \leq_{s} F$.

It is easy to see that this definition includes Horty's result model as the special case where for all cases the rule equals the case's fact situation. The counterpart of Proposition 6 can easily be obtained for this reason model by defining the relevant differences between a precedent and a focus case in Definition 9 relative to the precedent's rule instead of to its fact situation.

Definition 11 (Differences between cases with dimensions: reason model with complete rules.) Let $c=(F(c), R(c)$, outcome $(c))$ and $f=(F(f)$, outcome $(f))$ be two cases. The set $D(c, f)$ of differences between $c$ and $f$ is defined as follows. 
1. If outcome $(c)=$ outcome $(f)=s$ then $D(c, f)=\left\{(d, v) \in R(c) \mid v(d, c) \not \Varangle_{s} v(d, f)\right.$.

2. If outcome $(c) \neq$ outcome $(f) \quad$ whe re outcome $(c)=s$ th e n $D(c, f)=\left\{(d, v) \in R(c) \mid v(d, c) \Varangle_{\bar{s}} v(d, f)\right.$.

Proposition 8 Let, given a set $D$ of dimensions, $C B$ be a case base and $F$ a fact situation. Then deciding $F$ for $s$ is forced given $C B$ according to Definition 10 iff there exists a case in $C B$ with outcome s such that $D(c, f)=\emptyset$ according to Definition 11, where $f=(F, R, s)$ for some $R$.

Proof As for Proposition 6 with $F(c)$ replaced by $R(c)$.

Note that because of this result, Definition 10 does not have to quantify over cases with different rules as in Definition 5.

Since Bench-Capon and Atkinson (2017), Horty $(2019,2020)$ and Rigoni (2018) all discuss the merits of their models in terms of the fiscal-domicile example introduced by Prakken and Sartor (1998), I will from now on use that example as a running example. The issue is whether the fiscal domicile of a person who moved abroad for some time has changed. Let us consider two dimensions $d_{1}$, the duration of the stay abroad in months and $d_{2}$ the percentage of the tax-payer's income that was earned abroad during the stay. For both values, increasingly higher values increasingly favour the outcome change and decreasingly favour the outcome no change. So, for instance, $\left(d_{1}, 12 m\right)<_{\text {change }}\left(d_{1}, 24 m\right)$ and so $\left(d_{1}, 24 m\right)<_{\text {no change }}\left(d_{1}, 12 m\right)$. An example of a fact situation is $F=\left\{v\left(d_{1}\right)=30 \mathrm{~m}, v\left(d_{2}\right)=60 \%\right\}$ and an example of a case is $c=\left(F^{\prime}\right.$, change $)$ where $F^{\prime}=\left\{v\left(d_{1}\right)=12 m, v\left(d_{2}\right)=60 \%\right\}$.

This example can be used to show that Definition 10 does not collapse into the dimension-based result model. Suppose that $c$ has a fact situation $\left\{\left(v\left(d_{1}\right)=30 m\right),\left(v\left(d_{2}\right)=60 \%\right)\right\}$ and outcome change and consider again fact situation $F=\left\{\left(v\left(d_{1}\right)=24 m\right),\left(v\left(d_{2}\right)=75 \%\right)\right\}$. Suppose the court in $c$ ruled that with a percentage earned abroad of $60 \%$ a stay abroad of at least 12 months suffices for change of fiscal domicile. The rule of $c$ then is $\left\{\left(d_{1}, 12 m\right),\left(d_{2}, 60 \%\right)\right\}$. Then in the reason model deciding $F$ for change is forced, even though the stay abroad in $F$ is shorter than in $c$, so even though $F$ is weakened for the outcome along that dimension, since the stay is still longer than its value in $c$ 's rule. By contrast, in the result model this difference suffices to make $c$ distinguishable and deciding $F$ for no change allowed.

The model of Definition 10 also avoids an arguably counterintuitive feature of Horty's (2019) dimension-based model (which Horty (2017) also himself noted and which he avoids in his revised (2020) model). In the tax example, if the rule of $c$ is $\left\{\left(d_{1}, 12 m\right)\right\}$ then in a new case in which the stay abroad is 24 months and the percentage of income earned abroad is 75\% deciding for change is in Horty's model not forced by the precedent, since it is weaker for change than the precedent in that the stay abroad is not 30 but 24 months. However, this seems counterintuitive given that the court in the precedent ruled that 12 months abroad suffice for change and given that the new case is stronger for this outcome in its only 
other dimension. With Definition 10 deciding for change is instead forced by $c$, since $\left\{\left(d_{1}, 12 m\right),\left(d_{2}, 60 \%\right)\right\} \leq_{\text {change }}\left\{\left(d_{1}, 24 m\right),\left(d_{2}, 75 \%\right)\right\}$.

One issue remains. Horty's factor-based reason model requires that courts select a rule in the new case that leaves the case base consistent when the case is added to it. In Horty's model consistency is defined in terms of a preference relation between sets of reasons pro and con a decision (cf. Definition 3 above). However, the present model does not distinguish between pro and con value assignments, while still a notion of consistency is needed. Consider again the tax example with the two dimensions $d_{1}$ and $d_{2}$ and consider two precedents $c_{1}$ with rule $R_{1}=\left\{\left(d_{1}, 12 m\right),\left(d_{2}, 60 \%\right)\right\}$ and outcome change and $c_{2}$ with rule $R_{2}=\left\{\left(d_{1}, 8 m\right),\left(d_{2}, 60 \%\right)\right\}$ and outcome no change. Consider next a fact situation $F$ with $d_{1}=15$ and $d_{2}=60 \%$. Then deciding $F$ for change is forced. Suppose the court does so but formulates the rule $R_{3}=R_{2}=\left\{\left(d_{1}, 7 m\right),\left(d_{2}, 60 \%\right)\right\}$. Then in a new fact situation equal to rule $R_{2}$ both deciding change and deciding no change would be forced, so adding $f=\left(F, R_{3}\right.$, change $)$ would make it inconsistent in that for the same fact situation two opposite outcomes are forced. So a constraint on rule selection should be that it should leave a consistent case base consistent in this sense.

\subsection{A reason model with dimensions with switching points and incomplete rules}

The second way in which the result model can be refined into a reason model is by allowing that the rule $R$ of a case assigns a value to a subset of its fact situation $F_{D}$, while still adhering to the constraint that the rule's values of dimensions are at most a favourable to the case's decision as their actual values in the case. How can precedential constraint then be defined? If for each relevant value assignment in a case it is known which side is favoured by it, that is, if each such assignment is turned into a factor, then this is relatively easy, by combining ideas of Rigoni (2018) and Horty (2019). As a matter of fact, neither of these papers contains such a combination, although it seems implicitly assumed by Rigoni. So the present section can be regarded as a full formalisation and a formal exploration of Rigoni's ideas, although restricted to his special case of so-called 'complete' dimensions, which have a fixed 'switching point' and a total ordering on its values, which are all known.

Unlike Horty, Rigoni assumes that each dimension has a switching point, which is a subset of 'adjacent' values, to one side of which every value assignment favours one side and to the other side of which every value assignment favours the other side. Then Rigoni extracts 'dimensional factors' from dimensions, which like 'standard factors' favour one side or another except that they are not just given (as standard factors are) but derived from dimensions. I formalise these ideas as follows.

Let $o$ and $\overline{o^{\prime}}$ be two outcomes. A dimension with a switching point is a tuple $d=\left(V, \leq_{s}, \leq_{o^{\prime}}, \operatorname{Pro}(d), \operatorname{Con}(d)\right)$ where $V, \leq_{o}$ and $\leq_{o^{\prime}}$ are as before and Pro $(d)$ and $\operatorname{Con}(d)$ are disjoint subsets of $V$ such that

- for all $v \in \operatorname{Pro}(d)$ and $v^{\prime} \notin \operatorname{Pro}(d)$ it holds that $v^{\prime}<_{o} v$;

- for all $v \in \operatorname{Con}(d)$ and $v^{\prime} \notin \operatorname{Con}(d)$ it holds that $v^{\prime}<{ }_{o^{\prime}} v$; 
- for all $v, v^{\prime} \in V:$ if $v \in \operatorname{Pro}(d)$ and $v \leq_{o} v^{\prime}$ then $v^{\prime} \in \operatorname{Pro}(d)$;

- for all $v, v^{\prime} \in V$ : if $v \in \operatorname{Con}(d)$ and $v \leq_{o^{\prime}} v^{\prime}$ then $v^{\prime} \in \operatorname{Con}(d)$.

This implicitly defines a dimension's switching point $S(d)$ as $V \backslash \operatorname{Pro}(d) \cap \operatorname{Con}(d)$. Note that the definitions imply that for all $v \in S, v^{\prime} \in \operatorname{Pro}(d)$ and $v^{\prime \prime} \in \operatorname{Con}(d)$ it holds that $v<_{o} v^{\prime}$ and $v<_{O^{\prime}} v^{\prime}$. Thus the definitions formalise Rigoni's idea that the switching point consists of all values that are 'between' the pro- $O$ and pro- $O$ ' values. Note that any of the sets $S, \operatorname{Pro}(d)$ and $\operatorname{Con}(d)$ can be empty. This allows to capture HYPO-style dimensions that 'generally favour a particular side', namely, by leaving $S$ and one but not both of $\operatorname{Pro}(d)$ and $\operatorname{Con}(d)$ empty. Finally, given a set $D$ of dimensions with switching points, fact situations can now be partial in that they can consist of value assignments to any nonempty subset of $D$. For any fact situation $F$ we say that $F^{\prime} \subseteq F$ favours $s$ iff either $s=o$ and $F^{\prime} \subseteq\{(v, d) \mid d \in D$ and $v \in \operatorname{Pro}(d)\}$ or $s=o^{\prime}$ and $F^{\prime} \subseteq\{(v, d) \mid d \in D$ and $v \in C o n(d)\}$. For any fact situation $F$, the set $F^{s}$ is the subset of all value assignments in $F$ favouring side $s$.

In our tax example, we could have the following three dimensions, with $o=$ change.

$d_{1}=$ duration; $V=$ any number of months in natural numbers; $v \leq_{o} v^{\prime}$ iff $v \leq v^{\prime} ; v \in \operatorname{Pro}\left(d_{1}\right)$ iff $v \geq 18 ; v \in \operatorname{Con}\left(d_{1}\right)$ iff $v \leq 6$

$d_{2}=$ earned; $V=$ any percentage in natural numbers; $v \leq_{o} v^{\prime}$ iff $v \leq v^{\prime}$; $v \in \operatorname{Pro}\left(d_{2}\right)$ iff $v \geq 60 ; v \in \operatorname{Con}\left(d_{2}\right)$ iff $v \leq 30$.

$d_{3}=$ gave up house; $\quad V=\{$ yes, no $\} ; \quad$ no $<_{o}$ yes $; \quad \operatorname{Pro}\left(d_{3}\right)=\{$ yes $\}$; $\operatorname{Con}\left(d_{3}\right)=\{n o\}$.

Given a set $D$ of dimensions with switching points as just defined, a case $c$ is now a tuple $(F, \operatorname{pro}(c), \operatorname{con}(c), s)$, where $F$ is a fact situation, pro $(c)$, the case's reason, is a value assignment to a subset of $D$ such that for each $(d, v) \in \operatorname{pro}(c)$ there exists a $\left(d^{\prime}, v^{\prime}\right) \in F^{s}$ such that $d=d^{\prime}$ and $v \leq_{s} v^{\prime}$, and $\operatorname{con}(c)=F^{\bar{s}}$. This notion of the reason of a case is essentially the same as the one of Horty's factor-based reason model, in that it consists of a subset of all factors in the case favouring its outcome. The only difference is that now the factors favouring the two outcomes are not simply given but derived from a specification of dimensions with switching points. Then, just as for the factor-based reason model, we can say that a case decision expresses a preference for the case's reason pro(c) over its set $\operatorname{con}(c)$. It is left to formally define this case-related preference relation. Horty (2019) in fact defines such a preference relation, but on a different notion of the reason of a case, which I will briefly discuss below in Sect. 5.4. For now I simply adapt Horty's definition to the just defined notion of a reason.

Let $(d, v)$ and $\left(d, v^{\prime}\right)$ be two value assignments both favouring side $s$. Then $(d, v)$ entails $\left(d, v^{\prime}\right)$ iff $v^{\prime} \leq_{s} v$. Generalising, a set $X$ of value assignments favouring $s$ entails a value assignment $(d, v)$ favouring $s$ iff some value assignment in $X$ entails $(d, v)$, and a set $X$ of value assignments favouring $s$ entails a set $Y$ of value assignments favouring $s$ iff each value assignment in $Y$ is entailed by a value assignment in $X$. Note that for each case the set $F^{s}$ entails the case's reason $\operatorname{pro}(c)$. Then Definition 3 can be adapted to the case with dimensions as follows: 
Definition 12 Let $C B$ a case base given a set $D$ of dimensions with switching points and consisting of cases $c=(F, \operatorname{pro}(c), \operatorname{con}(c), s)$, and let $X$ and $Y$ be sets of value assignments favouring $\bar{s}$ and $s$, respectively. Then for every every $c \in C B$ :

1. $Y<_{c} X$ iff $\operatorname{con}(c)$ entails $Y$ and $X$ entails pro(c);

2. $Y<_{C B} X$ iff $Y<_{c} X$ for some $c \in C B$.

Definition 4 then applies provided the superset relation between factor sets is replaced by the entailment relation between sets of value assignments. Then Definition 5 can be applied to sets of value assignments as follows.

Definition 13 (Precedential constraint with dimensions with a switching point: a reason model with possibly incomplete rules) Let, given a set $D$ of dimensions, $C B$ be a case base in which all cases have a rule and $F$ a fact situation. Then, given $C B$, deciding $F$ for $s$ is forced iff for all cases $c=(F, \operatorname{pro}(c), \operatorname{con}(c)$, outcome $(c))$ such that $C B \cup\{c\}$ is consistent it holds that outcome $(c)=s$.

To show that this reason model does not collapse into the result model, consider in our running example a case $c$ with fact situation $\left\{\left(v\left(d_{1}\right)=30 m\right),\left(v\left(d_{2}\right)=75 \%\right),\left(v\left(d_{3}\right)=n o\right)\right\}$ and reason $\left\{v\left(d_{1}\right)=24 m\right\}$ and outcome change, and a new fact situation $F=\left\{\left(v\left(d_{1}\right)=27 m\right),\left(v\left(d_{2}\right)=50 \%\right),\left(v\left(d_{3}\right)=n o\right)\right\}$. Then deciding $F$ for change is forced in the reason model but not in the result model. In the reason model the outcome of $c$ expresses that $\left\{v\left(d_{3}\right)=n o\right\}<_{c}\left\{v\left(d_{1}\right)=24 m\right\}$. Moreover, we have that $v\left(d_{1}\right)=27 \mathrm{~m}$ entails $v\left(d_{1}\right)=24 \mathrm{~m}$, so we have $\left\{v\left(d_{3}\right)=n o\right\}<_{c}\left\{v\left(d_{1}\right)=27 m\right\}$. By contrast, in the result model deciding $F$ for change is not forced since, firstly, $v\left(d_{1}, F\right)=27 m<_{\text {change }} v\left(d_{1}, c\right)=30 m$ and, secondly, $v\left(d_{2}, F\right)=50 \%<_{\text {change }} v\left(d_{2}, c\right)=75 \%$.

The counterpart of Proposition 8 can be obtained for this adapted reason model by again redefining the relevant differences between a precedent and a focus case, now also taking missing and new dimension-based factors into account. Essentially, this definition combines the factor-based Definition 6 with the dimension-based Definition 11.

Definition 14 (Differences between cases with dimensions with switching points and incomplete rules) Let $c=(F(c)$,pro $(c), \operatorname{con}(c)$,outcome $(c))$ and $f=(F(f), \operatorname{pro}(f), \operatorname{con}(f)$, outcome $(f))$ be two cases. The set $D(c, f)$ of differences between $c$ and $f$ is defined as follows.

1. If outcome $(c)=$ outcome $(f)=s$ then $D(c, f)=\{(d, v) \in \operatorname{pro}(c) \mid v(d, f) \in \operatorname{pro}(f)$ and $\left.v(d, c) \not_{s} v(d, f)\right\} \cup\{(d, v) \in \operatorname{pro}(c) \mid v(d, f) \notin \operatorname{pro}(f)\} \cup\{(d, v) \in \operatorname{con}(f) \mid$ $v(d, f) \notin \operatorname{con}(c)\}$.

2. If outcome $(c) \neq$ outcome $(f) \quad$ whe r e outcome $(c)=s \quad$ th e $\mathrm{n}$ $D(c, f)=\left\{(d, v) \in \operatorname{pro}(c) \mid v(d, f) \in \operatorname{con}(f)\right.$ and $\left.v(d, c) \Varangle_{\bar{s}} v(d, f)\right\} \cup\{(d, v) \in$ $\operatorname{pro}(f) \mid v(d, f c \notin \operatorname{con}(c)\} \cup\{(d, v) \in \operatorname{pro}(c) \mid v(d, f) \notin \operatorname{con}(f)\}$. 
Proposition 9 Let, given a set $D$ of dimensions with switching points, $C B$ be a case base and $F$ a fact situation. Then deciding $F$ for $s$ is forced given $C B$ according to Definition 13 iff there exists a case in $C B$ with outcome s such that $D(c, f)=\emptyset$ according to Definition 14, where $f=\left(F, F^{s}, F^{\bar{s}}, s\right)$.

Proof Assume first that deciding $F$ for $s$ is forced. Then every case $f^{\prime}=\left(F, \operatorname{pro}\left(f^{\prime}\right), F^{s}, \bar{s}\right)$ is inconsistent with the case base. Let without loss of generality $\operatorname{pro}\left(f^{\prime}\right)=F^{\bar{s}}$. Then since $C B$ is consistent, by Observation 1 of Horty (2019) there exists a case $c=\left(F, \operatorname{pro}(c), F^{\bar{s}}, s\right) \in C B$ such that $\operatorname{pro}(c)<_{f^{\prime}} F^{\bar{s}}$ and $F^{\bar{s}}<_{c} \operatorname{pro}(c)$.

That pro $(c)<_{f^{\prime}} F^{\bar{s}}$ implies that $\operatorname{con}\left(f^{\prime}\right)$ entails $\operatorname{pro}(c)$. But $\operatorname{con}\left(f^{\prime}\right)=F^{s}$, so then $F^{s}$ entails $\operatorname{pro}(c)$. Now consider any $\left(d, v_{c}\right) \in \operatorname{pro}(c)$. Then there exists a $(d, v) \in F^{s}$ such that $v_{c} \leq_{s} v$. So the first set on the right-hand side of condition (1) of Definition 14 is empty. Moreover, that $F^{s}$ entails $\operatorname{pro}(c)$ also implies that there does not exist a $(d, v) \in c$ such that $d$ does not have a value in $F^{s}$. So the second set on the righthand side of condition (1) of Definition 14 is also empty. Finally, that $F^{\bar{s}}<{ }_{c}$ pro(c) implies that $\operatorname{con}(c)$ entails $\operatorname{con}(f)$. So the third set on the right-hand side of condition (1) of Definition 14 is also empty. So $D(c, f)=\emptyset$.

Assume next that there exists a $c \in C B$ with outcome $s$ and such that $D(c, f)=\emptyset$. Then $F^{\bar{s}}<_{c} \operatorname{pro}(c)$ and since $D(c, f)=\emptyset$ we also have that pro $(f)$ entails $\operatorname{pro}(c)$. But then $F^{\bar{s}}<_{c} \operatorname{pro}(f)$, so for every $X$ implied by $F^{\bar{s}}$ we have $X<_{c} \operatorname{pro}(f)$. Any reason for deciding $F$ for $\bar{s}$ requires adding a case $c^{\prime}=\left(F, \operatorname{pro}\left(c^{\prime}\right), F^{s}, \bar{s}\right)$ to $C B$. But then $F^{s}<_{c}^{\prime} \operatorname{pro}\left(c^{\prime}\right)$, so also $\operatorname{pro}(f)<_{c}^{\prime} \operatorname{pro}\left(c^{\prime}\right)$. But $\operatorname{pro}\left(c^{\prime}\right)$ is implied by $F^{\bar{s}}$, so we have $\operatorname{pro}\left(c^{\prime}\right)<_{c} \operatorname{pro}(f)$, so $C B$ is inconsistent.

This completes the formalisation and investigation of Rigoni's (2018) dimensionbased account of precedential constraint with complete dimensions.

\subsection{An alternative dimension-based reason model}

While the reason model of the previous section is well-behaved, it has an important pragmatic limitation, since it requires that for each dimension it is defined in general, abstracting from specific cases, whether a particular value assignment favours a side and if so, which side it favours. The problem is that, unlike with factors, it may in practice be hard for knowledge engineers to identify the side favoured by a particular value assignment, since often this will be context-dependent. See also Bench-Capon (1999) and Rissland and Ashley (2002); the latter remark at p. 69 that the direction of a value assignment is not for the knowledge engineer to decide but for the judge in a case.

Consider, for example, in our tax example a case with fact situation $\left\{v\left(d_{1}\right)=30 m, v\left(d_{2}\right)=60 \%\right\}$ and with outcome change. Are both value assignments pro this outcome, or is one pro and the other con change? And if the latter, then which is pro and which is con? This is not easy to say in general. On the other hand, what is uncontroversial is that increasingly higher values for these dimensions increasingly support change and decreasingly support no change. In 
Horty's $(2019 ; 2020)$ approach this problem arises to a lesser extent, since he works with so-called magnitude factor expressions, which are of the form 'the actual value of dimension $d$ in the case favours side $s$ at least as much as reference value $p^{\prime}$; this expression is meant to favour side $s$. Such expressions are, unlike Rigoni's switching points, not part of the general specification of dimensions but of individual case formulations, so they do not need to be chosen in general. Yet in this approach the problem still arises to some extent. For instance, in the just-given example still a reference value has to be known, as well as a side favoured by it for both dimensions. Such information may be hard to identify in case decisions.

For these reasons I will in this subsection explore an alternative approach, in which all that is needed is general knowledge about which side is favoured more and which side less if a value of a dimension changes, as captured by the two partial orders $\leq_{s}$ and $\leq_{s^{\prime}}$ on a dimension's values. These partial orders will usually be obvious from the domain. Of course, a potential downside of my alternative approach is that it sacrifices some expressivity of Horty's approach, as I will further illustrate below after defining the approach.

Dimensions and cases are now again defined as in Sect. 5.2. Below for any two sets $X$ and $Y$ of value assignments, $Y^{\mid X}$ is the subset of $Y$ that consists of value assignments to any dimension that is also assigned a value in $X$.

Definition 15 (Precedential constraint with dimensions: an alternative reason model with possibly incomplete rules) Let, given a set $D$ of dimensions, $C S$ be a case base in which all cases have a possibly incomplete rule and $F$ a fact situation. Then deciding $F$ for $s$ is forced iff there exists a case $c=\left(F^{\prime}, R, s\right)$ in $C B$ such that $R \leq_{s} F^{\mid R}$

So deciding $F$ for $s$ is forced iff there is a precedent for $s$ such that $F$ is at least as favourable for $s$ on all dimensions in the precedent's rule.

Moreover, like with the reason model with complete rules, the constraint on rule selection is needed that adding a new case to a consistent case base should leave the case base consistent in that for no fact situation two opposite outcomes are forced.

To see how this definition works, consider again the tax example with dimensions $d_{1}$ and $d_{2}$ and consider precedent $c$ with fact situation $\left\{\left(v\left(d_{1}\right)=30 m\right),\left(v\left(d_{2}\right)=60 \%\right)\right\}$, with rule $R=\left\{\left(d_{1}, 12 m\right)\right\}$ and with outcome change. Consider next a fact situation $F$ with $v\left(d_{1}\right)=24 m, v\left(d_{2}\right)=50 \%$. Then deciding $F$ for change is forced since $F^{\mid R}=\left\{\left(d_{1}, 24 m\right)\right\}$ and we have that $R=\left\{\left(d_{1}, 12 m\right)\right\}<_{\text {change }}\left\{\left(d_{1}, 24 m\right)\right\}$. Note that deciding $F$ for change is forced by $c$ even though $F$ is in both dimension weaker for change than $c$. The point is that with respect to $d_{1}$ the rule declares a stay of 12 months sufficient and that $d_{2}$ is not in $c$ 's rule.

Since a rule that assigns a value to all dimensions in $D$ is a special case, the above example that shows that Definition 10 does not collapse into the dimension-based result model also holds for this definition. It is also easy to show 
that Definition 15 just as its counterpart for cases with complete rules avoids the above-mentioned counterintuitive consequences. Moreover, a counterpart of Proposition 6 can be obtained for this reason model by redefining the relevant differences between a precedent and a focus case as follows. They are now relative to the cases' rules instead of to its $F_{D}$, and differences can now only be relevant on dimensions that are assigned values in both cases.

Definition 16 (Differences between cases with dimensions and possibly incomplete rules) Let $c=(F(c), R(c)$, outcome $(c))$ and $f=(F(f), R(f)$, outcome $(f))$ be two cases. The set $D(c, f)$ of differences between $c$ and $f$ is defined as follows.

\section{Ifoutcome $(c)=$ outcome $(f)=\operatorname{sthen} D(c, f)=\left\{(d, v) \in F(f)^{\mid R(c)} \mid v(d, c) \not \Varangle_{s} v(d, f)\right.$ \\ 2. If outcome $(c) \neq$ outcome $(f) \quad$ wh e r e $\quad$ outcome $(c)=s \quad$ t h e $\mathrm{n}$ $D(c, f)=\left\{(d, v) \in F(f)^{\mid R(c)} \mid v(d, c) \nsupseteq_{\bar{s}} v(d, f)\right.$.}

Clause (1) says that if the outcomes of the precedent and the focus case are the same, then any value assignment in the focus case to a dimension in the precedent's rule that is not at least as favourable for the outcome as in the precedent is a relative difference. Clause (2) says that if the outcomes are different, then any value assignment in the focus case to a dimension in the precedent's rule that is not at most as favourable for the outcome of the focus case as in the precedent is a relative difference. Thus any value assignment in the focus case to a dimension that is not in the rule's precedent is irrelevant for whether the precedent constrains the focus case, but all value assignments in the focus case to dimensions in the precedent's rule are relevant for this question, regardless of whether these value assignments are in the focus case's rule.

Proposition 10 Let $C B$ be a case base given a set $D$ of dimensions in which all cases have a complete rule and let $F$ be a fact situation. Then deciding $F$ for $s$ is forced given $C B$ according to Definition 15 iff there exists a case $c=(F(c), R(c), s)$ in $C B$ such that $D(c, f)=\emptyset$, where $f=(F, R(f), s)$ for any $R(f)$.

Proof As for Proposition 6 with $F(c)$ replaced by $R(c)$ and $F(f)$ replaced by $F(f)^{\mid R(c)}$.

On the other hand, this approach also has limitations. Since it does not allow to indicate for a particular value assignment that it favours a particular outcome, some fine-grained distinctions in Horty's and Rigoni's approaches between cases where a decision is, or is not forced cannot be made. Consider again the last example. We saw that deciding fact situation $F$ for change was forced by precedent $c$ even though $F$ is weaker for change than $c$ on dimension $d_{2}$, since $d_{2}$ is not in $c$ 's rule. This might be regarded a problem since the fact that the percentage of income earned abroad was less in the new situation $F$ than in the precedent might by a decision maker be regarded as an exception to the precedent's rule. In Rigoni's approach as formalised 
above this can be formalised by letting, for instance, all values of $d_{2}$ greater than or equal to $60 \%$ be the switching point, so that every value less than $60 \%$ favours no change. Then $d_{2}=50 \%$ in $F$ is a relevant difference with precedent $c$ and deciding for change is not forced.

Or consider a variant of this example with the same precedent $c$ with $v\left(d_{1}\right)=30 m, v\left(d_{2}\right)=60 \%$, with outcome change and with rule $\left\{\left(d_{1}, 12 m\right),\left(d_{2}, 60 \%\right)\right\}$, but with a focus case $f$ with $v\left(d_{1}\right)=24 m, v\left(d_{2}\right)=75 \%$. Next, consider a third dimension $d_{3}$ on whether the tax payer kept his original house or gave it up, with the ordering kept $<_{\text {change }}$ gave up, and suppose that in $c$ we have $v\left(d_{3}\right)=$ gave up while in $f$ we have $v\left(d_{3}\right)=$ kept. Then $f$ is weaker for change on $d_{3}$ than $c$. Yet according to Definition 10 precedent $c$ cannot be distinguished and deciding $f$ for change is forced. In Rigoni's approach as formalised above, $v\left(d_{3}\right)=$ gave up can be defined to be a factor favouring no change, and then $v\left(d_{3}\right)=$ gave up is a relevant difference between $c$ and $f$.

It seems that the only sensible way to change the definition to allow $c$ to be distinguished is to allow any value assignment to any dimension not mentioned in $c$ 's rule to be a relevant difference, since we cannot indicate whether such a value assignment favours a particular outcome.

Let us discuss this in more general terms. In the factor-based reason model the idea of a rule has a clear intuition, namely, that the pro-decision factors in the rule are sufficient to outweigh the con-decision factors in the case. However, with dimensions, where it cannot always be said that a value assignment favours a particular side, this intuition does not apply, since the value assignments outside the rule do not necessarily favour the opposite outcome. All that can said is that by stating the rule the court has decided that, given the rule, the case's value assignments to the other dimensions are irrelevant. The question then is whether such a ruling is defeasible. If it is not, then every new case in which the dimensions in the precedent's rule have values that are at least as favourable to the decision as in the rule is constrained by the precedent regardless of possible differences on the other dimensions. If that is regarded as too rigid, then there are two options. The first is that value assignments to dimensions not in a precedent's rule can be a reason for distinguishing just in case in the new fact situation they are less favourable for the precedent's outcome than in the precedent. But then the model collapses into the reason model with complete rules. The second option is that every value assignment to a dimension that is not in the rule of the case can override the case's outcome. In other words, every such value assignment can be an exception to the original ruling that the dimensions outside the rule are irrelevant. But then a problem similar to the problem with Horty's (2019) reason model arises: in our last example any income percentage, even a percentage higher than $60 \%$, would suffice to distinguish $c$. A possible solution to this problem might be refining the model by allowing but not requiring to indicate that a particular value assignment favours a particular outcome. Then in the last example, indicating that $60 \%$ is a pro-change assignment would suffice to prevent $c$ from being distinguished on higher percentages. I leave the development of this idea to future research. For now it can be concluded that a Rigoni-style approach in which value assignments are always pro a particular outcome leads to finer-grained distinctions between 
forced and not-forced decisions than the present approach but is arguably harder to apply in practice (although this should be verified in realistic applications). To a lesser extent this also holds for a Horty-style approach with magnitude factors.

\section{Combining factors and dimensions}

I now turn to the question how factors and dimensions can be combined in a single model of precedential constraint. It would at first sight seem that factors are simply a special case of dimensions with just two values 0 and 1 where $0<<_{s} 1$ while $1<_{\bar{s}} 0$. However, upon closer inspection this is not the case, since with factors there is more to say than just that the two sides have opposed preferences over the presence or absence of a factor. Consider, for example, in the tradesecrets domain the factor bribed. That the defendant bribed one of the plaintiff's employees surely is a factor pro misuse of trade secrets, but that the defendant did not bribe any of the plaintiff's employees does not have to be regarded as a factor con that outcome: it can also be regarded as neutral with respect to that outcome. Therefore, it makes sense to treat factors differently than dimensions.

Accordingly, I introduce some new terminology. Each two-valued dimension in $D$ comes with a partial function $t_{d}: V \longrightarrow\left\{o, o^{\prime}\right\}$ that assigns to zero, one or both values of the dimension an outcome subject to two constraints (henceforth if $d$ is two-valued and $v$ is one value of $d$ then $\bar{v}$ denotes the other value of $d$ ):

1. if $t_{d}(v)=o$ then $t_{d}(\bar{v})=o^{\prime}$ or $t_{d}(\bar{v})$ is undefined.

2. if $t_{d}(v)=o$ then $\bar{v}<_{o} v$.

The $t_{d}$ function captures which outcome is favoured by a value of $d$, if any. Any value assignment $(d, v)$ to a two-valued dimension $d$ such that $t_{d}(v)=o$ is called a pro- $o$ factor. The terminology of Sect. 3 also applies to such factors. Then $D^{t}$ is the subset of $D$ of two-valued dimensions for which $t_{d}$ is defined for at least one value, and $D^{m}=D \backslash D^{t}$. A dimensional fact set $F^{t}\left(F^{m}\right)$ assigns values to all dimensions in $D^{t}\left(D^{m}\right)$.

In our trade secrets example, we could have that $t_{d}(v)=\pi$ for deceived and bribed with value 1 and $t_{d}(v)=\delta$ for obtainable-elsewhere and reverse-eng with value 1 , and that in all other cases $t_{d}(v)$ is undefined.

It can be proven that if $D^{m}$ is empty, that is, we only have factors, then Definition 8 of the dimension-based result model of precedential constraint reduces to its factor-based counterpart. In what follows, for any set of dimensions $D$ with associated set of functions $t_{d}$, and for any dimensional fact situation $F$, let $F^{s}$ be $\left\{(d, x) \mid(d, x) \in F\right.$ and $\left.t_{d}(x)=s\right\}$. Furthermore, for any case base $C B$ given a set of dimensions $D$ with associated set of functions $t_{d}$ such that $D^{m}=\emptyset$ its factor-based counterpart $C B^{f}$ is obtained by replacing each $c=(F, s)$ in $C B$ with $c^{f}=\left(F^{s}, F^{\bar{s}}, s\right)$. Note that in the translation from $c$ to $c^{f}$ value assignments in $c$ that do not favour a side do not occur in $F^{s} \cup F^{\bar{s}}$. 
Proposition 11 Let $C B$ be a case base given a set of dimensions $D$ with associated set of functions $t_{d}$ such that $D^{m}=\emptyset$ and let $C B^{f}$ be its factor-based counterpart. Then deciding $F$ for $s$ is forced given $C B$ according to Definition 8 iff deciding $F^{s} \cup F^{\bar{s}}$ for $s$ is forced given $C B^{f}$ according to Definition 2.

Proof It suffices to prove for any pair of dimensional fact situations $X$ and $Y$ that (1) $X^{s} \subseteq Y^{s}$ and $Y^{\bar{s}} \subseteq X^{\bar{s}}$ iff (2) for all $(d, v) \in X$ and all $\left(d, v^{\prime}\right) \in Y$ it holds that $v(d) \leq_{s} v^{\prime}(d)$.

For the only-if part, suppose (1) and consider any $(d, v) \in Y^{s}$ such that $(d, v) \notin X^{s}$. Then $(d, v) \in Y$ while $(d, \bar{v}) \in X$. Moreover, by the second constraint on $t_{d}$ we have $\bar{v}<_{s} v$. Consider next any $(d, v) \in X^{\bar{s}}$ such that $(d, v) \notin Y^{\bar{s}}$. Then $(d, v) \in X$ while $(d, \bar{v}) \in Y$. Moreover, by the second constraint on $t_{d}$ we have $\bar{v}<_{\bar{s}} v$, so we have $v<_{s} \bar{v}$. Then (2) follows since $\leq_{s}$ and $\leq_{s}$ are partial orders.

The proof in the other direction is similar and omitted here.

\section{Adding definitions of precedential constraint to HYPO and CATO}

In this section I further discuss the HYPO and CATO systems in light of the present paper. Both systems have ways of establishing the presence or absence of factors (CATO) or value assignments to dimensions (HYPO) from more concrete factual information. The models of precedential constraint discussed in this paper can abstract from these ways, although they may be relevant for determining their input, for instance, to which extent a closed-world assumption should be applied to absent factors. Given this abstraction, CATO's factors conform to the format of this paper's factor-based models but in HYPO this is more subtle. As described by Ashley (1990), HYPO's knowledge structures are dimensions and a factor is defined as a dimension that applies in a case. So in HYPO the notion of a factor is case-related and a factor can be multi-valued, unlike in the line of research initiated by Horty (2011). Moreover, although HYPO's dimensions are initially defined as in Sect. 5.1 of the present paper, then the possibility is added to declare of a given dimension that it generally favours a particular side. Finally, HYPO does not make the assumption of this paper's Sect. 5 that all dimensions are assigned a value in each case.

As noted in the introduction, the work on HYPO and CATO was not concerned with precedential constraint but with generating arguments. Yet it is interesting to see whether the notions of precedential constraint discussed in this paper can be applied in these systems. In CATO the present factor-based models of Sect. 3 can be directly applied but in HYPO things are more complicated given the way it defines factors in terms of dimensions. In fact, HYPO has a definition of relevant differences between two cases that essentially combines the present Definitions 6 and 9 (Ashley 1990, p. 159). Slightly rephrased in this paper's notation:

Definition 17 (Differences between cases: $H Y P O$ ) Let $\mathcal{D}$ be a set of dimensions and $D^{s}$ and $D^{\bar{s}}$ be two disjoint subsets of $D$ of dimensions generally favouring, 
respectively, side $s$ and $\bar{s}$. A case $c$ is now a pair $c=(F(c)$, outcome $(c))$, where $F(c)$ is a partial dimensional fact situation, that is, a value assignment to a subset of $D$. For any case $c$ the set $\operatorname{pro}(c)$, respectively, $\operatorname{con}(c)$ is defined as the set of all dimensions assigned a value in $c$ that generally favour side $s$, respectively $\bar{s}$. Now let $c=(F(c), s)$ and $f=(F(f), s)$ be two cases with the same outcome. Then the set $D(c, f)$ of differences between $c$ and $f$ is defined as follows.

$$
\begin{aligned}
& D(c, f)=[\operatorname{pro}(c) \backslash \operatorname{pro}(f) \cup \operatorname{con}(f) \backslash \operatorname{con}(c)] \cup[\{(d \in D \mid v(d, c) \text { and } v(d, f) \text { are } \\
& \text { defined and } \left.v(d, c) \not_{s} v(d, f)\right]
\end{aligned}
$$

It is easy to that (1) if all dimensions are assigned a value in both cases, then the definition reduces to Definition 9 of dimension-based differences, while (2) if for all $d$ such that both $v(d, c)$ and $v(d, f)$ are defined it holds that $v(d, c) \leq_{s} v(d, f)$ then the definition reduces to Definition 6 of factor-based differences. This suggests that Definition 17 could be directly applied to define precedential constraint by defining that deciding $f$ for $s$ is forced given $C B$ iff there exists a case $c$ with outcome $s$ in $C B$ such that $D(c, f)=\emptyset$. It would then be interesting to find combinations of Definitions 2 and 8 corresponding to Definition 17. Note that HYPO's most-on-point relation between cases, which looks at the set-inclusion relation between the factor sets of cases, is not suitable for defining a notion of precedential constraint, since it disregards dimensions and also which side is favoured by a factor.

\section{Conclusion}

In this paper I have formally analysed some existing and new factor- and dimensionbased models of precedential constraint. I have made several technical contributions. I formalised how relevant differences between cases can be used to identify forced decisions, I showed that in some cases the difference between absent and negated factors does not matter, and I identified conditions under which factors equate twovalued dimensions. I also fully formalised Rigoni's 2018 dimension-based model of precedential constraint with complete dimensions. In addition, I argued that Rigoni's model and to a lesser extent Horty's $(2019,2020)$ dimension-based reason models have pragmatic limitations in that they require the specification of information that may be hard to obtain in practical applications. I proposed an alternative dimension-based reason model without this limitation, although also with lesser ability to distinguish between situations in which a decision is or is not forced by a body of precedents. Future research should focus on whether the limitations of the various models can be overcome without sacrificing their benefits. More generally, future research on precedential constraint could focus on incorporating richer representations of cases into the models, such as recordings of the court's arguments for their decisions. The vast body of existing work on legal case-based reasoning could be utilised for this purpose. Of course, there is the legal-theoretic issue whether the court's arguments for their decisions are legally binding, but a formal theory of precedential constraint can abstract from such legal issues. 
As for related research on precedential constaint, the above sections contain detailed comparisions with the recent studies of Horty and Rigoni. I now briefly discuss some other related research. In several papers, Trevor Bench-Capon has, either alone or with others, argued that dimensions cannot be reduced to factors (BenchCapon 1999; Bench-Capon and Rissland 2001; Bench-Capon and Atkinson 2017, 2018). Similar observations were made by Rissland and Ashley (2002). My account in Sect. 5.4 respects this, since it does not require that value assignments to dimensions are said to favour one side or another. In fact, as shown in Sect. 6, for both this model and Horty's dimension-based result model (discussed in Sect. 5.1), the converse also holds, namely, that factors cannot simply be reduced to two-valued dimensions (although we saw that properly making this distinction is still an open problem). Bench-Capon and Atkinson (2017) criticise Rigoni's account on the grounds that it cannot deal with questions of balance and trade off. I believe that both my model of Sect. 5.4 and Horty's dimension-based result model leave room for this but outside these models (likewise Horty 2020). Models of precedential constraint only address the problem of how a decision is constrained by a body of precedents; they do not address the problem of how to argue for particular outcomes in a new case that is not constrained by a body of precedents. I believe that the latter is where the relevance of balancing and trade off is located.

Open Access This article is licensed under a Creative Commons Attribution 4.0 International License, which permits use, sharing, adaptation, distribution and reproduction in any medium or format, as long as you give appropriate credit to the original author(s) and the source, provide a link to the Creative Commons licence, and indicate if changes were made. The images or other third party material in this article are included in the article's Creative Commons licence, unless indicated otherwise in a credit line to the material. If material is not included in the article's Creative Commons licence and your intended use is not permitted by statutory regulation or exceeds the permitted use, you will need to obtain permission directly from the copyright holder. To view a copy of this licence, visit http://creativecommons.org/licen ses/by/4.0/.

\section{References}

Aleven V (1997) Teaching case-based argumentation through a model and examples. PhD Dissertation, University of Pittsburgh

Aleven V, Ashley K (1991) Toward an intelligent tutoring system for teaching law students to argue with cases. In: Proceedings of the third international conference on artificial intelligence and law. ACM Press, New York, pp 42-52

Ashley K (1989) Toward a computational theory of arguing with precedents: accomodating multiple interpretations of cases. In: Proceedings of the second international conference on artificial intelligence and law. ACM Press, New York, pp 39-102

Ashley K (1990) Modeling legal argument: reasoning with cases and hypotheticals. MIT Press, Cambridge

Ashley K (2004) Case-based models of legal reasoning in a civil law context. In: Proceedings of the International Congress of Comparative Cultures and Legal Systems of the Instituto de Investigaciones Juridicas, Universidad Nacional Autonoma de Mexico, Mexico City

Bench-Capon T (1999) Some observations on modelling case based reasoning with formal argument models. In: Proceedings of the seventh international conference on artificial intelligence and law. ACM Press, New York, pp 36-42 
Bench-Capon T, Atkinson K (2017) Dimensions and values for legal CBR. In: Wyner A, Casini G (eds) Legal knowledge and information systems. JURIX 2017: the thirtieth annual conference. IOS Press, Amsterdam, pp 27-32

Bench-Capon T, Atkinson K (2018) Lessons from implementing factors with magnitude. In: Palmirani M (ed) Legal knowledge and information systems. JURIX 2018: the thirty-first annual conference. IOS Press, Amsterdam, pp 11-20

Bench-Capon T, Rissland EL (2001) Back to the future: dimensions revisited. In: Verheij B, Lodder A, Loui R, Muntjewerff A (eds) Legal knowledge and information systems. JURIX 2001: the fourteenth annual conference. IOS Press, Amsterdam, pp 41-52

Gardner A (1987) An artificial intelligence approach to legal reasoning. MIT Press, Cambridge

Hage J (1993) Monological reason-based logic: a low-level integration of rule-based reasoning and casebased reasoning. In: Proceedings of the fourth international conference on artificial intelligence and law. ACM Press, New York, pp 30-39

Horty J (2011) Rules and reasons in the theory of precedent. Leg Theory 17:1-33

Horty J (2017) Reasoning with dimensions and magnitudes. In: Proceedings of the sixteenth international conference on artificial intelligence and law. ACM Press, New York, pp 109-118

Horty J (2019) Reasoning with dimensions and magnitudes. Artif Intell Law 27:309-345

Horty J (2020) Modifying the reason model. Artif Intell Law. https://doi.org/10.1007/s10506-020-09275 $-\mathrm{Z}$

Horty J, Bench-Capon T (2012) A factor-based definition of precedential constraint. Artif Intell Law 20:181-214

Loui R, Norman J, Olson J, Merrill A (1993) A design for reasoning with policies, precedents, and rationales. In: Proceedings of the fourth international conference on artificial intelligence and law. ACM Press, New York, pp 202-211

Prakken H (2019) Comparing alternative factor- and precedent-based accounts of precedential constraint. In: Araszkiewicz M, Rodriguez-Doncel V (eds) Legal knowledge and information systems. JURIX 2019: the thirty-second annual conference. IOS Press, Amsterdam, pp 73-82

Prakken H, Sartor G (1998) Modelling reasoning with precedents in a formal dialogue game. Artif Intell Law 6:231-287

Rigoni A (2015) An improved factor based approach to precedential constraint. Artif Intell Law 23:133-160

Rigoni A (2018) Representing dimensions within the reason model of precedent. Artif Intell Law 26:1-22

Rissland E, Ashley K (1987) A case-based system for trade secrets law. In: Proceedings of the first international conference on artificial intelligence and law. ACM Press, New York, pp 60-66

Rissland E, Ashley K (2002) A note on dimensions and factors. Artif Intell Law 10:65-77

Publisher's Note Springer Nature remains neutral with regard to jurisdictional claims in published maps and institutional affiliations. 